\title{
Evolution of the Pax-Six-Eya-Dach network: the calcisponge case study
}

Sofia AV Fortunato ${ }^{1,2}$, Sven Leininger ${ }^{1,3}$ and Maja Adamska ${ }^{1 *}$

\begin{abstract}
Background: The Pax-Six-Eya-Dach network (PSEDN) is involved in a variety of developmental processes, including well documented roles in determination of sensory organs and morphogenesis in bilaterian animals. Expression of PSEDN components in cnidarians is consistent with function in sensory organ development. Recent work in demosponges demonstrated the presence of single homologs of Pax and Six genes, and their possible involvement in morphogenesis, but the absence of the remaining network components. Calcisponges are evolutionarily distant from demosponges, and the developmental toolkits of these two lineages differ significantly. We used an emerging model system, Sycon ciliatum, to identify components of the PSEDN and study their expression during embryonic and postembryonic development.

Results: We identified two Pax, three Six and one Eya genes in calcisponges, a situation strikingly different than in the previously studied demosponges. One of the calcisponge Pax genes can be identified as PaxB, while the second Pax gene has no clear affiliation. The three calcisponge Six genes could not be confidently classified within any known family of Six genes. Expression analysis in adult S. ciliatum demonstrated that representatives of Pax, Six and Eya are expressed in patterns consistent with roles in morphogenesis of the choanocyte chambers. Distinct paralogues of Pax and Six genes were expressed early in the development of the putative larval sensory cells, the cruciform cells. While lack of known photo pigments in calcisponge genomes precludes formal assignment of function to the cruciform cells, we also show that they express additional eumetazoan genes involved in specification of sensory and neuronal cells: Elav and Msi.
\end{abstract}

Conclusions: Our results indicate that the role of a Pax-Six-Eya network in morphogenesis likely predates the animal divergence. In addition, Pax and Six, as well as Elav and Msi are expressed during differentiation of cruciform cells, which are good candidates for being sensory cells of the calcaronean sponge larvae.

Keywords: Calcisponges, Sycon, Eyes absent, Pax, Six, Sensory cells

\section{Background}

In insect and vertebrate model systems, Pax, Six, Eyes $a b$ sent (Eya) and Dachshund (Dach) form a network (PSEDN) interconnected by a series of protein-protein and proteinDNA interactions $[1,2]$. This network is often referred to as Retinal Determination Gene Network (RDGN), although it is involved in a variety of developmental processes in addition to eye development, including roles in morphogenesis of other sensory organs, kidneys and the branchial arches [3-5]. It has been suggested that the insect and vertebrate PSEDN/RDGN are not homologous [6], although studies in cnidarians indicate deep evolutionary roots of

\footnotetext{
* Correspondence: maja.adamska@sars.uib.no

'Sars International Centre for Marine Molecular Biology, University of Bergen, Thormøhlensgt. 55, Bergen 5008, Norway

Full list of author information is available at the end of the article
}

this network. In particular, it has been demonstrated that members of the Pax, Six and Eya families are expressed during neural development and sensory organ formation in a wide range of cnidarians [7-15]. In demosponges, two components of the PSEDN have been identified, PaxB and Six1/2 [16,17]. A recent study in the freshwater demosponge Ephydatia muelleri shows that these genes are co-expressed, potentially interact and are likely involved in juvenile/adult morphogenesis [16,17].

While sponges lack a nervous system, larvae of some species have well defined sensory cells, organized into simple organ-like structures $[18,19]$. For example, the parenchymella-type larvae of Amphimedon queenslandica have a pigmented ring equipped with long "steering" cilia at their posterior pole known as the sensory organ of the larva. Although opsin is not found in the A. queenslandica 
genome, the larval phototactic behavior [20] is likely mediated by cryptochrome [21], which has also been suggested to participate in light reception in adult tissue of another demosponge, Suberites domuncula [22]. Significantly, AmqCry2 expression is associated with the pigment ring [23]. Unfortunately, no information regarding expression of Pax or Six genes during development of the pigment ring is published, making it impossible to predict whether the ancestral PSEDN function was related to morphogenesis only or both morphogenesis and sensory organ formation.

We have recently began developing Sycon ciliatum as a model representing calcisponges (subclass Calcaronea), a lineage evolutionarily distant from demosponges and appearing to significantly differ from demosponges in its gene content [24-27]. Embryonic and postembryonic development of syconoid calcaronean species is well studied, allowing us to relate gene expression patterns to developmental events. Importantly, different stages of radial (choanocyte) chamber morphogenesis can be compared in a single specimen fixed during the growth phase: when the asconoid body plan of the juvenile gives rise to the syconoid body plan of the adult, radial chambers form around the original central choanocyte chamber and continue to develop sequentially from bottom to top, with the region just under the osculum remaining in asconoid organization [28]. The amphiblastulae larvae of calcisponges from the subclass Calcaronea are strikingly different from the parenchymellae $[25,29,30]$. Amphiblastulae are composed of only three cell types of embryonic origin: macromeres, micromeres, and four cruciform cells distributed around the "equator" and conveying unique tetra-radial symmetry to the larva $[24,31]$. While the macromeres and micromeres participate in formation of the juvenile body upon metamorphosis, the cruciform cells degenerate upon settlement [32]. The function of cruciform cells has not been studied experimentally, but based on ultrastructure examination it has been suggested that they might act as photoreceptors [33]. Intriguingly, differentiating cruciform cells of S. ciliatum express SoxB [24], a transcription factor involved in bilaterian neurogenesis $[34,35]$ and expressed in cnidarian neurosensory cells [36]. In addition, they express several genes which, while clearly having multitude of roles in animal development, are also implicated in specification of neuronal cell types in eumetazoans: components of the Wnt pathway ( $d v l, t c f$ and beta-catenin), Smad1/5 and nanos, lending support to the notion that they could be sensory cells [25,37-39].In this study we chose to address the evolution of the PSEDN by studying expression of potential components of this network in S. ciliatum, focusing on the cruciform cells as the likely sensory cells of the larvae, and on the adult morphogenesis represented by formation of the radial (choanocyte) chambers.
We searched the genomic and transcriptomic datasets of S. ciliatum and a second calcaronean species, Leucosolenia complicata, for genes encoding the components of the PSED network. To gain additional insight into identity of the cruciform cells, we also searched for genes encoding known proteins involved in photoreception (opsin and cryptochrome), and the RNA binding proteins Elav and Musashi, which are involved in specification of neurosensory cells in eumetazoans. In this paper, we report that calciponge genomes contain an ortholog of the Eya gene, which has not been previously reported in demosponges. We have not identified opsin and dachshund in calcisponges, which is consistent with the absence of these genes in demosponges. On the other hand, cryptochrome, which is present in demosponges, and likely responsible for light perception in the demosponge larvae, is absent from the calcisponge genomes. Expression of Pax, Six, Eya, Msi and Elav genes in Sycon ciliatum was studied by in situ hybridization. Here we show that Pax, Six and Eya genes are coexpressed during morphogenesis of the radial chambers, and that Pax and Six, as well as Elav and Msi are coexpressed during formation of cruciform cells.

\section{Methods}

Sequence retrieval, alignment and phylogenetic analyses Sycon ciliatum and Leucosolenia complicata Pax, Six and Eya genes were identified by BLAST searches of Sycon and Leucosolenia draft genomes (a preliminary draft of Leucosolenia) and transcriptomes as previously described [24] using specific domains from the following taxa: Bilateria, Branchiostoma floridae and Mus musculus; Porifera, Amphimedon queenslandica; Cnidaria, Nematostella vectensis.

For Eya, alignments were performed using the conserved ED domain. For Pax genes two alignments were performed: in the first alignment, the complete paired domain (PD) was included and in the second alignment the truncated RED-PD domain was used. Lack of homeodomains in the calcisponge Pax sequences precludes homeodomain-based phylogenetic analyses. For Six genes, the homeodomain along with the extended sine oculis domain was used. A combination of ClustalX and MUSCLE was used for the alignments, which were manually corrected where necessary.

The amino acid substitution model of protein evolution was determined by ProtTest 3.0 [40]. For all analyses the best model of protein evolution was $\mathrm{LG}+\mathrm{G}$, except for the analysis of the complete PD domain of Pax genes where invariant site gamma $\mathrm{LG}+\mathrm{G}+\mathrm{I}$ was optimal. Bayesian and maximum likelihood (ML) analyses were undertaken on conserved regions. For the MrBayes 3.1 analyses [41] (with LG model incorporated by in-house modification), a set of four independent Metropoliscoupled Markov Chain Monte Carlo (MCMC) were 

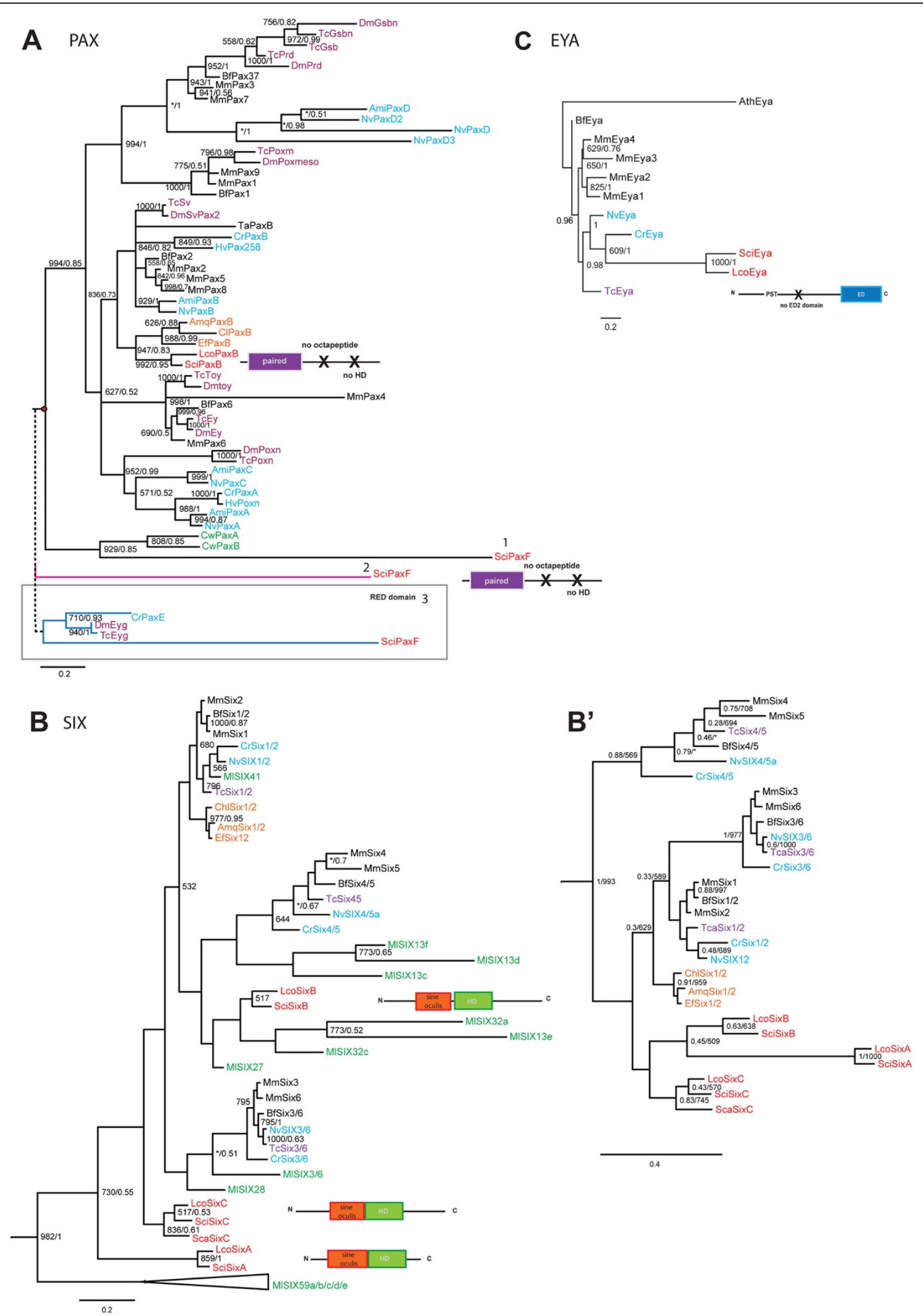

Figure 1 (See legend on next page.) 
(See figure on previous page.)

Figure 1 Phylogenetic analyses of the Six, Eya and Pax genes. A, Bayesian phylogenetic tree of Pax genes inferred from the paired domain. The red dots indicate the different positions of SciPaxF: 1, position of SciPaxF when the complete the PD domain was used to infer the phylogeny; 2, position of PaxF when removing ctenophore sequences and 3, when using the RED domain (S6) and including CrPaxE and Arthropod Eyg genes. B, The maximum likelihood tree was inferred from the Six homeodomain. The Six tree was rooted using the TALE class homeodomain as an outgroup. B', Bayesian tree inferred from the Six homeodomain without including Mnemiopsis Six genes. C, Bayesian tree of Eya ED domain. The EYA tree was rooted using Arabidopsis thaliana (At) Eya-like gene. ML bootstrap values greater than 500 (left) and posterior probabilities generated by MrBayes greater than 0.5 (right) are displayed. Asterisks indicate the differences in the position of a given gene. Names are prefixed as follows: Porifera: Calcisponges, $\mathrm{Sca}=$ Sycon calcavaris; $\mathrm{Sci}=$ Sycon ciliatum and Lco = Leucosolenia complicata. Demosponges, Amq = Amphimedon queenslandica; Ef = Ephydatia fluviatilis; $\mathrm{Chl}=$ Chalinula loosanoffi. Ctenophora: $\mathrm{Ml}=$ Mnemiopsis leidyi and Cw= Coeloplana willeyi. Cnidaria: Anthozoa, Nv = Nematostella vectensis and Hydrozoa $\mathrm{Cr}=$ Cladonema radiatu; $\mathrm{Hv}=$ Hydra vulgaris; Ami = Acropora millepora. Bilateria: Protostomia, Tc = Tribolium castaneum.; $\mathrm{Dm}=$ Drosophila melanogaster. Deuterostomia: Bf = Branchiostoma floridae and Mm = Mus musculus.

sampled every $1,000^{\text {th }}$ generation. Two Bayesian analyses were run for each dataset from 1 to 10 million generations, depending on the dataset. Convergence was assessed by plotting the log likelihood against the number of generations using Tracer v1.4 [42]. The analysis were stopped when the split frequency between the two runs was lower than 0.01 . After the removal of an appropriate burn-in (20 to $25 \%$ in most cases), the consensus trees were visualized with FigTree v1.4.0 [43]. The ML analysis was performed using PhyMl 3.0 [44] as follows: To provide a starting tree for the bootstrap analysis, two rounds of PhyMl analysis, each starting from five random trees, were run using the following command line: $-\mathrm{i}$ align.phy $-\mathrm{d}$ aa $-\mathrm{f}$ e $-\mathrm{m}$ LG $-\mathrm{c} 4-\mathrm{a}$ value $-v$ value - rand_start $-s$ NNI. The better of the two resulting ML trees (the tree with better likelihood value) was selected as an input tree for 1,000 bootstrap analysis using the following command line: $-\mathrm{i}$ align.phy $-\mathrm{d}$ aa $-\mathrm{f}$ e -m LG -c 4 -a value - $v$ value - u best_random_tree. nwk -b 1,000 -s NNI.

\section{Molecular analyses: RT-PCR and in situ hybridization}

Sample collection, fixation, PCR amplification of genes, sequencing, probe production and in situ hybridization in sliced sponges containing different reproductive stages and in small adult Sycon ciliatum specimens were performed as described previously [24].

\section{Results}

Two Pax genes are found in Sycon and one in Leucosolenia Two Pax genes were found in Sycon and one in Leucosolenia. As the Leucosolenia dataset is less extensive than the Sycon one, it is possible that our current analysis can miss a Leucosolenia sequence. In contrast to the demosponges' $\mathrm{PaxB}$ with a recognizable partial homeodomain and an octapeptide, the $\operatorname{Pax}$ genes in calcisponges do not appear to contain a homeodomain or octapeptide. Both Sycon Pax genes contain an intron in the PD domain (Additional file 1) corresponding to the intronexon boundaries found in cnidarians [45] and in other sponges $[16,46]$. The phylogenetic analysis of the PD of Pax (Figure 1C, Additional file 2) shows that both calcisponges have a single ortholog of $\operatorname{PaxB}$ and confirms the affiliation of demosponges $\operatorname{PaxB}$ genes as previously reported $[16,46]$. Several phylogenetic analyses were performed to determine affiliation of the second Pax gene in Sycon (Figure 1A). The first analysis used an alignment of the complete PD domain (Figure 1A, position 1), and indicated affiliation of this gene with the ctenophore Coeloplana willeyi Pax genes [47]. For the second analysis we removed the $C$. willeyi sequences from the alignment, and in this analysis the second Sycon Pax gene did not affiliate with any subfamily of Pax genes (Figure 1A, position 2). Finally, in the third analysis (Figure 1A, position 3 ), we used the partial PD domain (RED motif) and included arthropod eye gone (Eyg) genes and Cladonema radiatum PaxE gene. The result of the analysis shows that this second $\operatorname{Pax}$ gene fell within the PaxE subfamily (the Eyg subfamily) but this association was not supported (see also Additional file 3). Due to the unclear affiliation of the second Pax gene in Sycon, we decided to name it PaxF, following the next letter in the classification of Pax genes. Importantly, in none of our analyses SciPaxF affiliated with Pax1/9 and/or Pax3/7 subfamilies and thus it does not provide additional support for the notion of Pax duplication before divergence of Porifera [48].

\section{SINE class family is expanded in calcisponges}

We found three Six genes in each of the Sycon and Leucosolenia genomes corresponding to the SINE class of homeobox genes. All of them had the characteristic Six homeodomain with lysine at position 50 and the sine oculis DNA binding domain situated at the $-\mathrm{N}$ terminal to the homeodomain (Additional file 4), as seen in previously classified Six genes [49]. Additionally, we found two genes (SciHD35531and LcoHD71216) that contained partial sine oculis domains and homeodomains which displayed similarity to both the TALE and SINE gene classes. The homeodomains of SciHD35531 had a four amino acid insertion, instead of the three typically observed in TALE homeodomains (Additional file 5).

The first phylogenetic analysis was based on the homeodomain sequences and included the entire expanded 


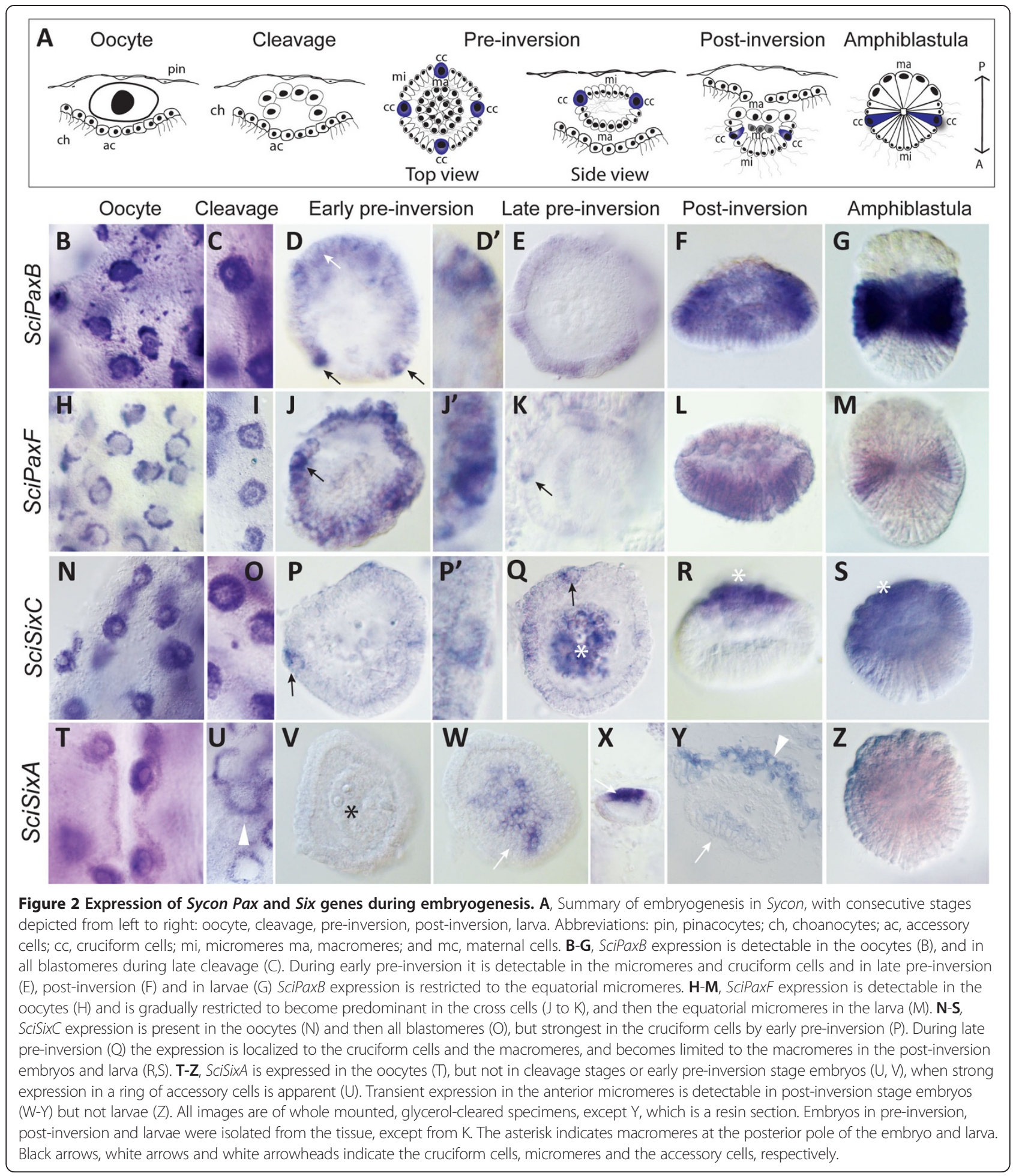

complement of ctenophore Six genes [50] (Figure 1B). This analysis correctly separated the cnidarian and bilaterian sequences into the three recognized families (Six1/2, Six $3 / 6$ and Six $4 / 5$ ) and confirmed the affiliation of demosponge Six genes within the Six1/2 family $[16,17,46]$ albeit with low support value (Figure 1B). Among the ctenophore sequences, MlSix41 nested within the Six $1 / 2$ family, consistent with the presence of the ETSY motif in its homeodomain, while MlSix36 and MlSix28 grouped with the Six $3 / 6$ family as described [50]. Surprisingly, none of the calcisponge sequences associated with demosponge sequences or any other recognized Six 
families. Because of unclear affiliation, calcisponge Six genes are referred to as $\operatorname{SixA}, \operatorname{Six} B$ and $\operatorname{SixC}$ (with the $\operatorname{Six} C$ sequences being most similar to the $\operatorname{SixC}$ sequence from Sycon calcaravis, ScaSixC [47]). In the tree presented on Figure 1B, Sci/LcoSixB genes associated with several of the ctenophore sequences, and this grouping receives moderate support in the ML analysis; while SciSixA and SciSixC fell completely outside of the recognized Six families. Given the difficulty in assigning the ctenophore Six genes and the fact that many of them are on long branches, we suspected that affiliations of calcisponge and Mnemiopsis Six genes might represent an artefact of long-branch attraction. We have thus carried out additional analyses with limited complements or completely without the ctenophore sequences, utilizing either only the homeodomain (Figure 1B' and Additional file 6), or the SINE domain together with the homeodomain (Additional file 7). In particular, we were hoping to differentiate between scenarios in which the three calcisponge Six genes are all descended from a Six 1/ 2 ancestral sequence, and are a result of family expansion in the calcisponge lineage, or are remnants of ancestral sequences, which are preserved in ctenophore and

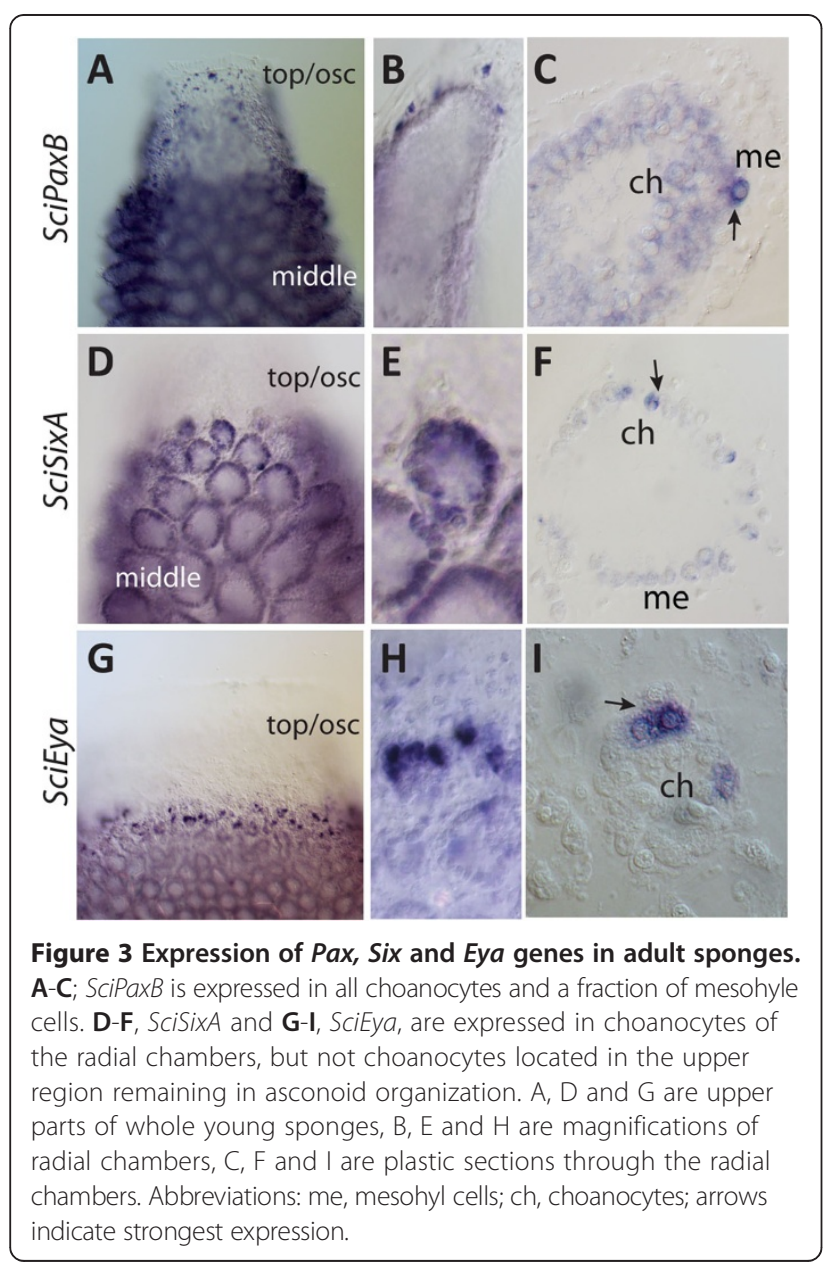

calcisponge genomes and lost in all others. In the homeodomain-only analysis without the ctenophore sequences, the calcisponge sequences clustered together, although this grouping did not receive significant support (Figure 1B'). In the tree utilizing the SINE domain, the calcisponge sequences did not form a monophyletic clade, but all of them branched basally (Additional file 7). Significantly, the grouping of the demosponge Six genes with the Six1/2 family was sensitive to taxon sampling. However, all demosponge Six and the calcisponge SixB homeodomains have the characteristic Six1/2 family 'ETSY' motif, and SixC sequences have a similar 'ETNY' motif ([50] and Additional file 5). Altogether, the results of the phylogenetic analyses indicate that the calcisponge Six genes may represent a specific diversification of the SINE class in calcareous sponges, similar to the one observed in the ctenophores [50], although the exact relationships between the paralogs remain unclear.

\section{Eyes absent (Eya) is present in calcisponges}

We found single orthologs of the eyes absent gene, Eya, in the two calcisponges. The Eya gene has not been previously reported in demosponges. In both species, the predicted proteins have a conserved $\mathrm{C}$-terminal amino-acid domain, the Eya domain (ED) but not the N-terminal ED2 domain (Additional files 8 and 9). The phylogenetic analysis in Figure $1 \mathrm{C}$ shows that the calcisponge Eya genes affiliate with the cnidarian Eya genes with moderate support values. Eya gene containing the ED domain and $\mathrm{P} / \mathrm{S} / \mathrm{T}$ rich region is found in the choanoflagelate Monosiga brevicollis [13], indicating that Eya was lost in demosponges. The presence of the ED domain and the $\mathrm{P} / \mathrm{S} / \mathrm{T}$ region but not the ED2 region of the Eya gene corroborates the notion that the ED2 domain was established in the last common ancestor of bilaterians and cnidarians [13].

\section{Pax and Six genes, but not Eya are expressed during embryogenesis and in the larvae}

As described for multiple species of calcaronean sponges $[25,29,51]$ and schematically represented on Figure 2A, embryogenesis of Sycon takes place in the mesohyl. Symmetric cleavage followed by cell differentiation leads to formation of a cup-shaped embryo composed of three cell types: macromeres, micromeres and cross cells. Macromeres are cells which are large, non-ciliated and granular in appearance, located close to the choanocytes of the parent sponge. Numerous micromeres are small and ciliated and located closer to the pinacocytes. The four cruciform cells are similar in size to the macromeres and are symmetrically distributed among the micromeres on the equator of the embryo. The embryo undergoes inversion while it translocates to the radial chamber and the mature larva swims through the oscular opening. 


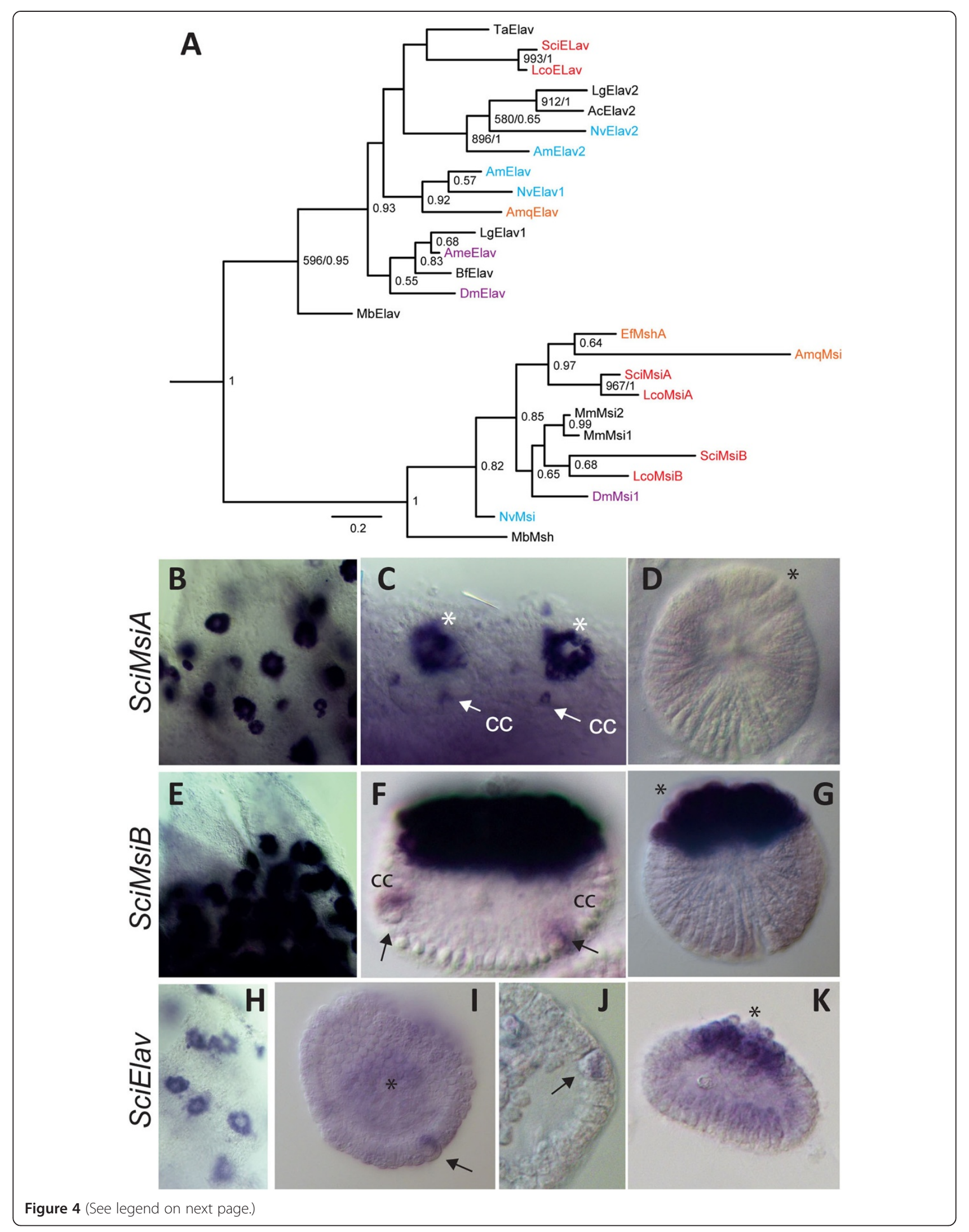




\section{(See figure on previous page.)}

Figure 4 Phylogenetic tree and embryonic expression of S. ciliatum Msi and Elav genes. A, This is a Bayesian tree inferred from the RRM2 motif of the RNAbp, Elav and Musashi. ML bootstrap values greater than 500 and posterior probabilities generated by MrBayes greater than 0.5 are displayed. The tree was rooted with the Pabp subfamily. Names are prefixed as in Figure 1 except from: Ac, Aplysia californica; Ame, Apis mellifera; Mb, Monosiga brevicollis; Lg, Lottia gigantea and Ta, Trichoplax adhaerens. B-K Whole mount in situ hybridization: B-D, SciMsiA expression in sponges containing oocytes (B); embryos during pre-inversion showing expression in the cruciform cells (cc, arrows) and macromeres (C) and no expression in the larva (D). E-G, SciMsiB expression in oocytes (E), in cc and macromeres in embryos during post-inversion (F), and expression in the macromeres in the larva (G). H-K, SciElav whole mount in situ of sponges containing oocytes (H); during pre-inversion SciElav is seen in cruciform cells and weak in macromeres $(\mathrm{I}, \mathrm{J})$; and by post-inversion SciElav expression is predominant in macromeres (K). The asterisk indicates macromeres at the posterior pole of the embryo and larva.

The expression of SciPaxB (Figure 2B-G) begins in the oocytes and continues during early and late cleavage, with transcripts present in all blastomeres (Figure 2C). During early pre-inversion, $S c i P a x B$ expression is evident in the cruciform cells and micromeres (Figure 2D, D'). In the larvae, SciPaxB expression is high in a broad band of equatorial micromeres (Figure 2F). The expression of SciPaxF begins in the oocytes (Figure $2 \mathrm{H}$ ) and is also initially uniform in all blastomeres, but by early preinversion the expression is stronger in micromeres and evident in the cruciform cells (Figure 2J-K). In the larvae, the SciPaxF expression is weak in the posterior micromeres, and expression in the cruciform cells is no longer apparent (Figure 2M). Expression of SciSixC is detected in the oocytes (Figure $2 \mathrm{~N}$ ), and during early pre-inversion it becomes most prominent in the cruciform cells (Figure 2P), resolving to strong expression in the macromeres and weaker in the cruciform cells during late pre-inversion (Figure 2Q). In post-inversion stages and larva, only macromere expression is apparent (Figure 2R, S).

The expression of SciSixA is strong in oocytes (Figure 2T), but not detectable in the cleavage stage embryos (Figure $2 \mathrm{U}-\mathrm{V}$ ), instead becoming prominent in a ring of accessory cells (which are derived from choanocytes) surrounding the embryos (Figure $2 \mathrm{U}, \mathrm{Y}$ ). In the late pre-inversion stage embryos, SciSixA expression is detectable in the anterior micromeres of the embryos (Figure 2W-Y) although this expression is no longer detectable in the larvae (Figure 2Z). Finally, the SciSixB gene expression was weak and appeared ubiquitous throughout all cell types with no distinct expression pattern (data not shown). No expression of SciEya was observed during embryogenesis, although its expression is detectable in choanocytes (Additional file 10 and see below).

\section{Pax-Six-Eya are co-expressed in choanocyte chambers}

In addition to the embryonic expression described above, SciPaxB, SciSixA and SciEya transcripts are detected in adult cell types, being most prominent in the choanocytes (the innermost epithelial cells responsible for feeding) (Figure 3). Expression of $S c i P a x B$ is uniform throughout the choanocytes and is strong in scattered mesohyl cells, particularly at the tips of the radial chambers and in the area of osculum (Figure 3A-C). SciSixA expression is stronger in choanocytes of the uppermost, forming chambers than in the older chambers, with somewhat heterogeneous expression among the choanocytes (Figure 3D-F). Notably, SciSixA expression is not detectable in choanocytes which are not organized in chambers, but located in the uppermost area of the sponge in the region of asconoid organization (Figure 3D). The expression of SciEya is similar to that of SciSixA in that the choanocytes of the oscular region are negative, the choanocytes located in the uppermost, forming choanocyte chambers display the most prominent expression, and the choanocytes of the older chambers display low level of expression (Figure 3G-I).

\section{Elav and Musashi are expressed in the cruciform cells and macromeres}

We identified one Elav and two Musashi genes in each of the two analyzed sponges (Additional file 11). The phylogenetic analyses were performed using the conserved RRM2 domain present in both gene families (Additional file 12). The calcisponge Elav genes affiliated with the Trichoplax adhaerens Elav gene, albeit with poor support. Affiliation of this clade with either Elav1 or Elav2 subfamily differed between ML and Bayesian analysis, never reaching significant support (Figure 4A). The calcisponge MsiA sequences clustered with demosponge $M s i$ genes, while the $M s i B$ pair affiliated with bilaterian sequences (Figure 4A).

Both of Msi genes and the Elav gene display similar expression patterns, being expressed strongly and uniformly in the oocytes and during cleavage, and gradually becoming most prominent in the cruciform cells (Figure 4B, E, $\mathrm{H}, \mathrm{I}$ and Additional file 13). During pre-inversion, strong expression of SciMsiA and weaker expression of both SciMsiB and SciElav is also detectable in the macromeres, in addition to continued expression in the cruciform cells (Additional file 13 and Figure 4C, J). Among the three genes, $S c i M s i B$ is detectable until the latest (post-inversion) stages in the cruciform cells (Figure 4F). Finally, SciMsiB and SciElav are detectable in the larval macromeres (Figure 4G, K). 


\section{Discussion}

Pax, Six, Eyes absent and Dachshund genes are involved in a variety of developmental processes, including the determination and morphogenesis of eyes and other sensory organs such as the ear [3,52]. In addition to overlapping expression patterns, protein-protein interactions and direct expression regulation demonstrate that PSED genes function as a network in a variety of animals [1,3]. Although many of the regulatory relationships are conserved in the PSED network, the interactions between network components can vary during development and in different animal lineages [6,53].

The origin of the PSED network has been previously investigated in non-bilaterian animals, including cnidarians $[7-9,13,54]$ and demosponges [16,47]. As demosponges contain PaxB and Six1/2 genes, but not Eya or Dach, it has been proposed that the ancestral network was composed of these two genes only. However, absence of Eya in demosponges appears secondary, as it is present in the choanoflagellates [13]. Here we show that the genomes of calcisponges also contain Eya, suggesting that its loss occurred in the lineage leading to demosponges and is thus not representative of all sponges. A question remains whether the three genes (Pax-Six-Eya) indeed do interact, and thus form a network, or whether the network arose later during evolution from components working individually in sponges. Importantly, a recent study in the demosponge Ephydatia mulleri indicated that in this species expression of Six1/2 might be controlled by $P a x B$ [17]. Unfortunately, it is not possible at the moment to functionally test interactions of the potential network components in calcisponges. However, their co-expression in several developmental contexts in Sycon suggests that these genes might also operate as a network, and in fact might control similar developmental processes as in other animals: sensory cell specification and morphogenesis. With the exception of Eya, all of the identified genes are co-expressed in the oocytes and in embryos undergoing cleavage, suggesting they are maternally expressed and have early developmental roles. In subsequent stages of development, the most striking and potentially informative co-expression is in the embryonic cruciform cells and later during adult morphogenesis, as summarized on Figure 5.

The cruciform cells have been suggested to act as larval photoreceptors [33] and the amphiblastulae have been shown to respond to light [55]. Surprisingly, we found that not only opsin, but also cryptochrome genes, which likely convey light sensitivity to the demosponge larvae, are absent from Sycon and Leucosolenia genomes, indicating that calcisponges have lost cryptochrome. Thus, calcisponge larvae might be able to detect light using another, yet unclear mechanism. Intriguingly, homologues of multiple genes involved in specification of
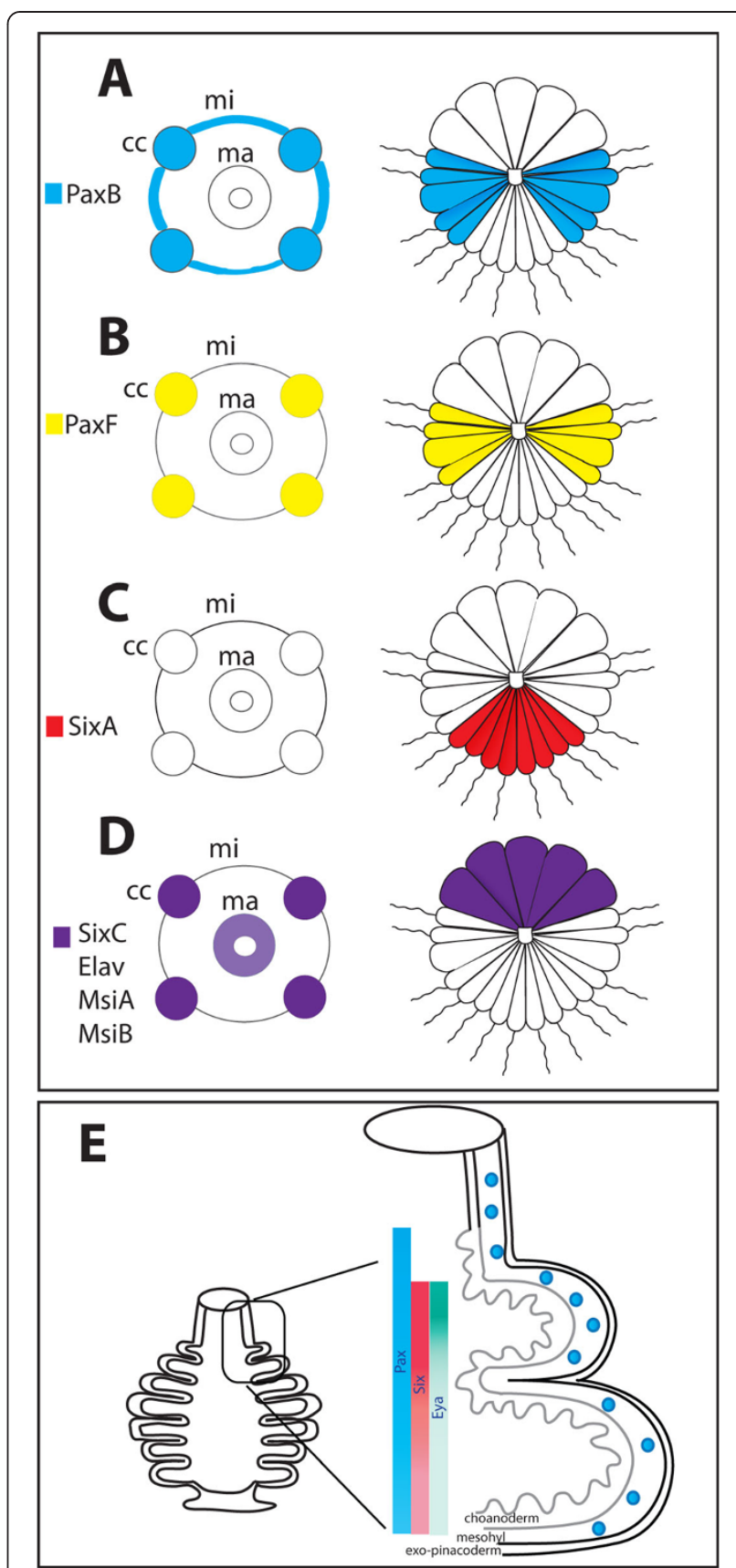

Figure 5 Summary of the expression patterns. A, SciPaxB in cruciform cells and equatorial micromeres during pre-inversion and micromeres in the larvae; $\mathbf{B}$, SciPaxF in cruciform cells during pre-inversion and equatorial micromeres of the larva; $\mathbf{C}$, SixA in anterior micromeres, D, SciSixC, SciElav, SciMsiA and SciMsiB in the cruciform cells and in the macromeres of the post-inversion stage embryos and/ or larvae; E, Gradients of SciSixA and SciEya in choanocyte cells organized in chambers, SciPaxB in all choanocytes and in scattered mesohyl cells.

neural and sensory cells in other animals are expressed during cross cell differentiation. These include $S c i P a x B$, SciPaxF, SciSixC, SciElav, SciMsiA and SciMsiB presented 
in this study (see Figure 5A), SciSoxB [24], components of the Wnt pathway (Dvl, Tcf and Beta-catenin), Smad1/5 and Nanos [25].

The observed co-expression indicates that SciSixC, Sci$P a x B$ and SciPaxF may potentially interact during embryogenesis, while SciPaxB, SciSixA and SciEya may potentially interact during adult morphogenesis (Figure 5). Among the three potential components of the PSEN in Sycon expressed in the choanocytes, $\operatorname{SciPaxB}$ has the broadest expression, with transcripts uniformly detected in all choanocytes. In contrast, SciSixA and SciEya transcripts are conspicuously absent from the uppermost choanocytes remaining in asconoid organization, but are particularly strongly expressed in the choanocytes of the uppermost chambers which are undergoing morphogenesis, with SciEya expression diminishing along a somewhat steeper gradient than SciSixA, so that both are expressed at low levels in the already formed chambers (Figure 5b). SciPaxB, SciSixA and SciEya expression patterns are thus consistent with interaction of these three genes during formation and maintenance of the organization of the radial chambers, and thus with a concerted role in morphogenesis. Despite lack of Eya of the demosponge Ephydatia, the morphogenetic role of the potential network might be a conserved feature, as knock down of EmPaxB and EmSix1/2 results in apparent dysmorphogenesis of the juveniles [17].

\section{Conclusions}

Overall, the presence of the sponge Eya gene and coexpression of Pax, Six and Eya genes in calcisponges indicate that the Pax-Six-Eya network may have already been established in the last common ancestor of sponges and eumetazoans, with Eya subsequently lost in demosponges. Based on gene expression during adult body plan formation in Sycon, we propose that this network had an ancient role in morphogenesis. Additionally, co-expression of Pax and Six with conserved eumetazoan neural genes Elav and $M s i$ in candidate larval sensory cells, suggests these genes could be ancestrally involved in the determination of sensory cell types. We envisage a scenario in which a simple PSE network was active in early metazoans, and that additional genes, such as Dachshund, were then later coopted into the network to expand its regulatory capacity in more complex animals.

\section{Additional files}

Additional file 1: The predicted Pax protein sequences and intron-exon boundaries. Exon-intron boundaries are highlighted in yellow, the paired domain is red.

Additional file 2: Alignment of the PD domain. This alignment was used for the phylogenetic analyses displayed in Figure $1 C$. The red box indicates the location of the RED motif used for the phylogenetic analyses in Additional file 3. Abbreviations are as in Figure 1.

Additional file 3: Bayesian phylogenetic tree of the Pax gene family inferred from the RED motif of the PRD domain. Support values on nodes are as follows: left, bootstrap (BT) values obtained from ML analysis; right, posterior probability from the Bayesian analysis. For abbreviations of species names see Figure 1.

Additional file 4: Six genes protein sequences in Sycon and Leucosolenia. Exon-intron boundaries are indicated by highlighting. Sine oculis domain is red, the homeodomain is underlined.

Additional file 5: Alignment of the homeodomain of the SINE and TALE classes including all of Six genes and selected TALE genes identified in calcisponges. Abbreviations are as in Figure 1.

Additional file 6: Maximum likelihood tree of the SINE class. Phylogenetic tree inferred from the homeodomain of Six and TALE genes. Bootstrap values are displayed on each node. Names are prefixed as in Figure 1. The tree was rooted with a selection of TALE class of homeobox genes. Mnemiopsis Six genes found in long branches on the tree from Figure 1B were not included in this analysis.

Additional file 7: Maximum likelihood phylogenetic analyses of sine oculis domain and homeodomain of the Six class. ML bootstrap values greater than 500 are displayed. Names are prefixed as in Figure 1.

Additional file 8: EYA protein sequences. Red indicates the location of the ED domain. Exon-intron boundaries are highlighted.

Additional file 9: Alignment of the ED domain. This alignment was used for the phylogenetic analyses displayed in Figure 1B. Abbreviations are as in Figure 1.

Additional file 10: The SciEya gene is not expressed during embryogenesis. A, oocytes; B, embryos during pre-inversion and C, post-inversion.

Additional file 11: Elav and Msi protein sequences. Exon-intron boundaries are highlighted. Red indicates the location of the RMM2 domain. Additional file 12: RRM2 motif alignment for Msi and Elav sequences. This alignment, without gaps, was used for the phylogenetic analyses displayed in Figure 4.

Additional file 13: Predominant expression of SciMsiA and SciElav in the cruciform cells. Late cleavage and pre-inversion stage embryos are shown for SciMsiA and SciElav, respectively.

\section{Abbreviations}

BLAST: Basic Local Alignment Search Tool; ED: Eya domain; ML: Maximum Likelihood; PD: Paired domain; PSEDN: Pax-Six-Eya-Dach network; RDGN: Retinal Determination Gene Network.

\section{Competing interests}

The authors declare that they have no competing interests.

\section{Authors' contributions}

SF and MA conceived and designed the study. MA and SL carried out field collection. SF performed phylogenetic analyses and analyzed expression of all genes presented in this manuscript except Musashi, which were studied by SL. SF drafted and MA edited the manuscript with input from SL. All authors read and approved the final manuscript.

\section{Acknowledgements}

We thank M. Adamski for sharing unpublished sequence assemblies and participation in field collections and G. Richards for insightful comments on the manuscript. This work was supported by the core budget of the Sars Centre to MA.

\section{Author details}

${ }^{1}$ Sars International Centre for Marine Molecular Biology, University of Bergen, Thormøhlensgt. 55, Bergen 5008, Norway. ${ }^{2}$ Department of Biology, University of Bergen, Thormøhlensgt. 55, Bergen 5008, Norway. ${ }^{3}$ Current address: Institute of Marine Research, Nordnesgaten 50, Bergen 5005, Norway. 
Received: 10 March 2014 Accepted: 15 May 2014

Published: 23 June 2014

\section{References}

1. Donner A, Maas R: Conservation and non-conservation of genetic pathways in eye specification. Int J Dev Biol 2004, 48:743-753.

2. Friedrich M: Ancient mechanisms of visual sense organ development based on comparison of the gene networks controlling larval eye, ocellus, and compound eye specification in drosophila. Arthropod Struct Dev 2006, 35:357-378.

3. Silver S, Rebay l: Signaling circuitries in development: insights from the retinal determination gene network. Dev Supp/ 2005, 132:3-13.

4. Kozmik Z, Holland N, Kreslova J, Oliveri D, Schubert M, Jonasova K, Holland L, Pestarino M, Benes V, Candiani S: Pax-Six-Eya-Dach network during amphioxus development: conservation in vitro but context specificity in vivo. Dev Biol 2007, 306:143-159.

5. Bassham S, Postlethwait JH: The evolutionary history of placodes: a molecular genetic investigation of the larvacean urochordate Oikopleura dioica. Development 2005, 132:4259-4272.

6. Wagner G: The developmental genetics of homology. Nat Rev Genet 2007, 8:473-479.

7. Matus D, Pang K, Daly M, Martindale M: Expression of Pax gene family members in the anthozoan cnidarian, Nematostella vectensis. Evol Dev 2007, 9:25-38

8. Suga H, Tschopp P, Graziussi DF, Stierwald M, Schmid V, Gehring WJ: Flexibly deployed Pax genes in eye development at the early evolution of animals demonstrated by studies on a hydrozoan jellyfish. Proc Natl Acad Sci U S A 2010, 107:14263-14268.

9. Kozmik Z, Daube M, Frei E, Norman B, Kos L, Dishaw L, Noll M, Piatigorsky J: Role of Pax genes in eye evolution: a cnidarian PaxB gene uniting Pax2 and Pax6 functions. Dev Cell 2003, 5:773-785.

10. Kumar J: The sine oculis homeobox (SIX) family of transcription factors as regulators of development and disease. Cell Mol Life Sci 2009, 66:565-583.

11. Kozmik Z: The role of Pax genes in eye evolution. Brain Res Bull 2008, 75:335-339.

12. Kozmik Z: Pax genes in eye development and evolution. Curr Opin Genet Dev 2005, 15:430-438.

13. Graziussi DF, Suga H, Schmid V, Gehring WJ: The "Eyes absent" (eya) gene in the eye-bearing hydrozoan jellyfish Cladonema radiatum: conservation of the retinal determination network. J Exp Zool B Mol Dev Evol 2012, 318:257-267.

14. Galliot B, Quiquand M, Ghila L, De Rosa R, Miljkovic-Licina M, Chera S: Origins of neurogenesis, a cnidarian view. Dev Biol 2009, 332:2-24.

15. Sinigaglia $C$, Busengdal $H$, Leclère $L$, Technau $U$, Rentzsch F: The bilaterian head patterning gene six3/6 controls aboral domain development in a cnidarian. PLoS Biol 2013, 11(2):e1001488.

16. Hill A, Boll W, Ries C, Warner L, Osswalt M, Hill M, Noll M: Origin of Pax and Six gene families in sponges: Single $P a x B$ and Six $1 / 2$ orthologs in Chalinula loosanoffi. Dev Biol 2010, 343:106-123.

17. Rivera A, Winters I, Rued A, Ding S, Posfai D, Cieniewicz B, Cameron K, Gentile $L$, Hill A: The evolution and function of the Pax/Six regulatory network in sponges. Evol Dev 2013, 15:186-196.

18. Maldonado M: The ecology of the sponge larva. Can J Zool 2006, 84:175-194.

19. Ludeman D, Farrar N, Riesgo A, Paps J, Leys S: Evolutionary origins of sensation in metazoans: functional evidence for a new sensory organ in sponges. BMC Evol Biol 2014, 14:3.

20. Leys S, Cronin T, Degnan B, Marshall J: Spectral sensitivity in a sponge larva. J Comp Physiol Neuroethol Sens Neural Behav Physiol 2002, 188:199-202.

21. Feuda R, Hamilton S, Mclnerney J, Pisani D: Metazoan opsin evolution reveals a simple route to animal vision. Proc Natl Acad Sci U S A 2012, 109:18868-18872.

22. Müller WE, Wang X, Schröder HC, Korzhev M, Grebenjuk VA, Markl JS, Jochum KP, Pisignano D, Wiens M: A cryptochrome-based photosensory system in the siliceous sponge Suberites domuncula (Demospongiae). FEBS J 2010, 277:1182-1201.

23. Rivera A, Ozturk N, Fahey B, Plachetzki D, Degnan B, Sancar A, Oakley T: Blue-light-receptive cryptochrome is expressed in a sponge eye lacking neurons and opsin. J Exp Biol 2012, 215:1278-1286.
24. Fortunato S, Adamski M, Bergum B, Guder C, Jordal S, Leininger S, Zwafink C, Rapp HT, Adamska M: Genome-wide analysis of the sox family in the calcareous sponge Sycon ciliatum: multiple genes with unique expression patterns. EvoDevo 2012, 3:14.

25. Leininger S, Adamski M, Bergum B, Guder C, Liu J, Laplante M, Bråte J, Hoffman NF, Fortunato S, Jordal S, Rapp HT, Adamska M: Developmental gene expression provides clues to relationships between sponge and eumetazoan body plans. Nat Commun 2014, 5:3905.

26. Riesgo A, Farrar N, Windsor PJ, Giribet G, Leys SP: The analysis of eight transcriptomes from all poriferan classes reveals surprising genetic complexity in sponges. Mol Biol Evol 2014, 31:1102-1120.

27. Sebé-Pedrós A, Ariza-Cosano A, Weirauch M, Leininger S, Yang A, Torruella G, Adamski M, Adamska M, Hughes T, Gómez-Skarmeta J, Ruiz-Trillo I: Early evolution of the T-box transcription factor family. Proc Natl Acad Sci U S A 2013, 110:16050-16055.

28. Maas O: Die Weiterentwicklung der Syconen nach der metamorphose. Zeutsch wiss Zool 1900, 67:215-240.

29. Ereskovsky AV: The Comparative Embryology of Sponges. Dordrecht Heidelberg London New York: Springer; 2010.

30. Maldonado M, Bergquist P: Atlas of Marine Invertebrate Larvae. San Diego, CA: Academic Press; 2002.

31. Manuel M: Early evolution of symmetry and polarity in metazoan body plans. C R Biol 2009, 332:184-209.

32. Amano S, Hori I: Metamorphosis of calcareous sponges. 2. Cell rearrangement and differentiation in metamorphosis. Invert Reprod Dev 1993, 24:13-26.

33. Tuzet O: Éponges calcaires. In Traité de Zoologie Anatomie, Systématique, Biologie Spongiaires. Edited by Grassé P-P. Paris: Masson et Cie; 1973:27-132.

34. Phochanukul N, Russell S: No backbone but lots of Sox: invertebrate Sox genes. Int J Biochem Cell Biol 2010, 42:453-464.

35. Watanabe H, Fujisawa T, Holstein TW: Cnidarians and the evolutionary origin of the nervous system. Dev Growth Differ 2009, 51:167-183.

36. Jager M, Queinnec E, Le Guyader H, Manuel M: Multiple Sox genes are expressed in stem cells or in differentiating neuro-sensory cells in the hydrozoan Clytia hemisphaerica. EvoDevo 2011, 2:12.

37. Kanska J, Frank U: New roles for Nanos in neural cell fate determination revealed by studies in a cnidarian. J Cell Sci 2013, 126:3192-3203.

38. Grigoryan T, Wend P, Klaus A, Birchmeier W: Deciphering the function of canonical Wnt signals in development and disease: conditional loss- and gain-of-function mutations of beta-catenin in mice. Genes Dev 2008, 22:2308-2341.

39. Hegarty S, O'Keeffe G, Sullivan A: BMP-Smad 1/5/8 signalling in the development of the nervous system. Prog Neurobio/ 2013, 109:28-41.

40. Abascal F, Zardoya R, Posada D: ProtTest: selection of best-fit models of protein evolution. Bioinformatics 2005, 21:2104-2105.

41. Ronquist F, Huelsenbeck JP: MrBayes 3: Bayesian phylogenetic inference under mixed models. Bioinformatics 2003, 19:1572-1574.

42. Rambaut A, Drummond AJ: Tracer v1.5.; 2009. http://beast.bio.ed.ac.uk/ software/tracer/.

43. Rambaut A: Figtree v1.4.0;; 2012. http://tree.bio.ed.ac.uk/software/figtree/,

44. Guindon S, Dufayard J-F, Lefort V, Anisimova M, Hordijk W, Gascuel O: New algorithms and methods to estimate maximum-likelihood phylogenies: assessing the performance of PhyML 3.0. Syst Biol 2010, 59:307-321.

45. Ryan J, Burton P, Mazza M, Kwong G, Mullikin J, Finnerty J: The cnidarianbilaterian ancestor possessed at least 56 homeoboxes: evidence from the starlet sea anemone. Nematostella vectensis. Genome Biol 2006, 7:R64.

46. Larroux C, Luke G, Koopman P, Rokhsar D, Shimeld S, Degnan B: Genesis and expansion of metazoan transcription factor gene classes. Mol Biol Evol 2008, 25:980-996

47. Hoshiyama D, Iwabe N, Miyata T: Evolution of the gene families forming the Pax/Six regulatory network: isolation of genes from primitive animals and molecular phylogenetic analyses. FEBS Lett 2007, 581:1639-1643.

48. Breitling R, Gerber JK: Origin of the paired domain. Dev Genes Evol 2000, 210:644-650.

49. Takatori N, Butts T, Candiani S, Pestarino M, Ferrier D, Saiga H, Holland P: Comprehensive survey and classification of homeobox genes in the genome of amphioxus, Branchiostoma floridae. Dev Genes Evol 2008, 218:579-590.

50. Ryan J, Pang K, Program NCS, Mullikin J, Martindale M, Baxevanis A: The homeodomain complement of the ctenophore Mnemiopsis leidyi suggests that Ctenophora and Porifera diverged prior to the ParaHoxozoa. EvoDevo 2010, 1:9. 
51. Franzen W: Oogenesis and larval development of Scypha Ciliata (Porifera, Calcarea). Zoomorphology 1988, 107:349-357.

52. Rebay I, Silver S, Tootle T: New vision from Eyes absent: transcription factors as enzymes. Trends Genet 2005, 21:163-171.

53. Schlosser G, Wagner GP: Modularity in development and evolution. Chicago: University of Chicago Press; 2004.

54. Stierwald M, Yanze N, Bamert R, Kammermeier L, Schmid V: The Sine oculis/Six class family of homeobox genes in jellyfish with and without eyes: development and eye regeneration. Dev Biol 2004, 274:70-81.

55. Elliot GR, Macdonald TA, Leys SP: Sponge larval phototaxis: a comparative study. Bollettino dei Musei e degli Istituti Biologici dell'Universita di Genova 2004, 68:291-300.

doi:10.1186/2041-9139-5-23

Cite this article as: Fortunato et al.: Evolution of the Pax-Six-Eya-Dach network: the calcisponge case study. EvoDevo 2014 5:23.

\section{Submit your next manuscript to BioMed Central} and take full advantage of:

- Convenient online submission

- Thorough peer review

- No space constraints or color figure charges

- Immediate publication on acceptance

- Inclusion in PubMed, CAS, Scopus and Google Scholar

- Research which is freely available for redistribution

Submit your manuscript at www.biomedcentral.com/submit
(O) Biomed Central 